\title{
Aspectos nutricionais na doença pulmonar
}

\author{
Nutritional aspects pulmonary disease
}

\author{
Katiele Baelz ${ }^{1}$, Cássia da Luz Goulart ${ }^{1}$, Andreia Lucia Gonçalves da Silva ${ }^{1}$, \\ Lisiane Lisboa Carvalho ${ }^{2}$, Fabiana Asmann Poll ${ }^{1,2}$
}

1 - Programa de Residência Multiprofissional no Hospital Santa Cruz, Santa Cruz do Sul, RS, Brasil.

2 - Universidade de Santa Cruz do Sul - UNISC, Santa Cruz do Sul, RS, Brasil.

fpoll@unisc.br

Palavras-chave:

Composição Corporal;

Doença Pulmonar Obstru-

tiva Crônica;Espirometria,

Micronutrientes.

\section{RESUMO}

Objetivos: associar o consumo alimentar, estado nutricional, composição corporal e os índices espirométricos em pacientes com doença pulmonar obstrutiva crônica (DPOC). Métodos: estudo transversal, com amostra de 27 pacientes com DPOC, segundo critérios do Global Iniciative for Chronic Obstructive Lung Disease (GOLD), de ambos os sexos, com idade igual ou superior a 50 anos. Aplicou-se o recordatório alimentar de 24 horas e realizou-se a adequação de consumo de macro e de alguns micronutrientes. A análise do estado nutricional e da composição corporal foi realizada através da bioimpedância elétrica, obtendo-se o índice de massa corporal (IMC), a massa gorda, massa livre e gordura. Para análise dos dados foram utilizadas medidas de tendência central e de dispersão. A comparação entre as variáveis numéricas, de acordo com o estado nutricional, foi realizada por meio da análise de variância para comparações múltiplas (ANOVA) com post hoc de Tukey. Foi considerado significativo um $\mathrm{p}<0,05$. Resultados: a idade média foi de $64,63 \pm 8,11$ anos, sendo $55,55 \%$ do sexo masculino, houve predomínio de obesidade, de ex-fumantes e estadiamento da doença grave (GOLD IV), segundo a estratificação do IMC. Quanto a composição corporal, pode-se constatar que o menor IMC e percentual de massa magra se relacionou com a gravidade da doença, observada na diminuição do volume expiratório forçado no primeiro segundo, com diferença estatisticamente significativa. $\mathrm{Na}$ análise dos micronutrientes, de um modo geral, os indivíduos obesos tiveram um consumo menor dos micronutrientes considerados, em comparação aos indivíduos eutróficos e desnutridos. Observou-se níveis diminuídos da ingestão alimentar de cálcio, magnésio em todos os estados nutricionais, sendo o paciente desnutrido com pior ingestão e pior função pulmonar (GOLD IV). Conclusão: alterações na composição corporal, essencialmente na massa magra, e na presença de desnutrição se associaram com maior gravidade da doença pulmonar, bem como se observou consumo inadequado de micronutrientes em todos os estados nutricionais, porém mais, na presença de obesidade.

\section{ABSTRACT}

Objectives: to associate food consumption, nutritional status, body composition and spirometric indices in patients with chronic obstructive pulmonary disease (COPD). Methods: cross-sectional study, with a sample of 27 COPD patients, according to the Global Initiative for Chronic Obstructive Lung Disease (GOLD) criteria, of both sexes, aged 50 or over. The 24 -hour food record was applied and the consumption of macro and some micronutrients was adjusted. Analysis of nutritional status and body composition was performed using electrical bioimpedance, obtaining the body mass index (BMI), fat mass, free mass and fat. For data analysis, measures of central tendency and dispersion were used. The comparison between the numerical variables, according to the nutritional status, was carried out through the analysis of variance for multiple comparisons (ANOVA) with Tukey's post hoc. $\mathrm{P}<0.05$ was considered significant. Results: the average age was $64.63 \pm 8.11$ years, $55.55 \%$ were male, with a predominance of obesity, ex-smokers and severe disease staging (GOLD IV), according to the stratification of BMI. As for body composition, the lowest BMI and percentage of lean mass were related to the severity of the disease, observed in the decrease in forced expiratory volume in the first second, with a statistically significant difference. In the analysis of micronutrients, in general, obese

Keywords: individuals had a lower consumption of the considered micronutrients, compared to eutrophic and malnourished individuals. Decreased levels of calcium and magnesium food intake were observed in all nutritional states, with malnourished patients presenting the worst intake andlung function (GOLD IV). Conclusion: changes in body composition, essentially in lean mass, and in the presence of malnutrition were associated with greater severity of lung disease, as well as inadequate consumption of micronutrients in all nutritional states, especially in the presence of obesity. 


\section{INTRODUÇÃO}

A Doença Pulmonar Obstrutiva Crônica (DPOC) acomete adultos acima dos 40 anos, com altas taxas de morbidade e mortalidade. ${ }^{1}$ Cerca de 210 milhões de pessoas no mundo têm DPOC e a estimativa é que se torne a terceira principal causa de morte por volta de $2020 .^{2}$

Estudos que avaliaram a associação da composição corporal e a função pulmonar em indivíduos com DPOC demonstram que alterações específicas e desfavoráveis na composição corporal, especialmente a diminuição da massa magra, podem ser preditores mais confiáveis de mortalidade do que somente o baixo Índice de Massa Corporal (IMC) de forma isolada. ${ }^{3-5}$ Sendo assim, a terapia nutricional nessa população é de suma importância devido ao seu grande impacto sobre a morbimortalidade da doença, visto que a redução do IMC é um fator de risco independente para mortalidade. A desnutrição na DPOC não depende de um único mecanismo, pois sua etiologia é multifatorial, sendo a ingestão inadequada de alimentos e o gasto energético aumentado os dois principais mecanismos envolvidos em sua gênese. ${ }^{6}$

Nesta população, alterações específicas e desfavoráveis na composição corporal, especialmente a diminuição da massa magra, pode ser o preditor mais confiável de mortalidade do que o baixo IMC de forma isolada. ${ }^{3,4}$ Essas alterações já foram relacionadas à intolerância ao exercício, comprometimento da qualidade de vida e aumento da mortalidade. ${ }^{7}$ Dessa forma, objetivou-se associar o consumo alimentar, o estado nutricional, a composição corporal e os índices espirométricos de pacientes com DPOC.

\section{MÉTODO}

Estudo de delineamento transversal de natureza quantitativa, aprovado pelo Comitê deÉtica em Pesquisa da Universidade de Santa Cruz do Sul sob o parecer $n^{\circ} 1.228 .408$. Foram incluídos no estudo 27 indivíduos com DPOC, diagnosticados segundo critérios do Global Iniciative for Chronic Obstructive Lung Disease (GOLD), ${ }^{8}$ de ambos os sexos, $\geq 50$ anos, clinicamente estáveis, sem exacerbações recentes e sem nenhum tipo de acometimento cardiovascular ou osteoarticular em acompanhamento pelo Programa de Reabilitação Pulmonar, desenvolvido em um hospital de ensino do interior do Rio Grande do Sul/RS.
Foram excluídos do presente estudo os pacientes com cardiopatias graves e que não possuíam avaliação espirométrica.

\section{Função pulmonar}

Foi avaliada por meio de um espirômetro digital (Microloop ${ }^{\circledR}$, MK8, Care Fusion, Germany) o volume expiratório forçado no primeiro segundo $\left(\mathrm{VEF}_{1}\right)$. A espirometria foi realizada de acordo com as recomendações da American Thoracic Society ${ }^{9}$ e os resultados analisados conforme os valores preditos de Pereira et al. ${ }^{10}$ A classificação da severidade da doença ${ }^{8}$ foi em moderado (GOLD II), severo (GOLD III) e muito severo (GOLD IV). Questionou-se o hábito de fumar atual ou anterior.

\section{Avaliação do consumo alimentar}

Aplicou-se o método do recordatório alimentar de 24 horas (R24h), com informações detalhadas sobre o consumo atual, número e horário das refeições realizadas no dia anterior ao da entrevista. ${ }^{11}$ Para análise de ingestão de macro e micronutrientes, foram utilizados os valores de ingestão recomendada (DRI's) para indivíduos do sexo masculino e feminino com faixa etária superior a 50 anos. ${ }^{12}$

A ingestão diária estabelecida para os macronutrientes em relação ao valor energético total foi de $55 \%$ para os carboidratos, 30\% para lipídios e $15 \%$ para proteínas. $^{13}$ Alguns micronutrientes específicos estudados para pacientes com DPOC, como ácido fólico, ferro, vitamina $\mathrm{C}$, vitamina $\mathrm{E}$, vitamina $\mathrm{A}$, cálcio, potássio, magnésio, fósforo e sódio tiveram sua ingestão analisada de acordo com o Estimated Average Requirement (EAR) das DRI's. ${ }^{12}$ A partir dessa análise, calculou-se a adequação do consumo dos nutrientes, e estabeleceu-se como faixa de normalidade valores entre 90 e $110 \% .^{14}$

\section{Avaliação da composição corporal e do estado nutricional}

O peso foi aferido por meio de uma balança mecânica calibrada (Filizola ${ }^{\circledR}$, Filizola S/A Pesagem e Automação, São Paulo, Brasil), e altura, por um estadiômetro acoplado a balança. O IMC foi determinado pela relação peso $(\mathrm{kg}) / \operatorname{altura}(\mathrm{m})^{2}$, seguindo os pontos de corte propostos pelo Nutrition Screening Initiative, a American Academyof Family Physicians e a American Dietetic Association, que sugerem valores de IMC $<22 \mathrm{~kg} / \mathrm{m}^{2}$ para desnutrição, IMC entre 22 e $27 \mathrm{~kg} / \mathrm{m}^{2}$ para eutrofia, e IMC $>27 \mathrm{~kg} /$ 
$\mathrm{m}^{2}$ para obesidade. ${ }^{15}$

A composição corporal dos pacientes foi avaliada por meio de bioimpedância elétrica de marca Biodynamics ${ }^{\circledR}$, modelo 420, versão 5.1 internacional. Os pacientes foram instruídos a respeitar as seguintes instruções: evitar consumo de álcool e cafeína (café, chá, chocolate) 24 horas antes do teste, não realizar atividade física intensa, não comer ou beber quatro horas antes da avaliação, suspender medicação diurética 24 horas antes, exceto no caso de indivíduos hipertensos, que devem estar sob rigoroso controle médico, não realizar exercícios físicos seis horas antes e esvaziar a bexiga 30 minutos antes do teste. Para execução do exame, o avaliado deveria estar em decúbito dorsal, numa posição confortável e relaxado, sem calçados, meias, relógio, pulseiras ou afins na mão direita. As pernas deveriam estar bem afastadas, as mãos abertas e apoiadas na maca. Foram colocados dois eletrodos no pé direito e dois eletrodos na mão direita. Os cálculos da composição corporal eram fornecidos de forma automática pelo próprio aparelho, com base nos dados inseridos pelo avaliador sobre altura, sexo e idade de cada paciente, e os dados de comparação ao esperado para o sexo foram fornecidos pelo fabricante. ${ }^{16}$ Foram considerados na presente pesquisa os resultados referentes à massa magra (kg e \%), gordura corporal (em Kg e \%), IMC $\left(\mathrm{kg} / \mathrm{m}^{2}\right)$, índice de massa livre de gordura (IMLG) e ângulo de fase.

Como parâmetro indicativo de sarcopenia foi utilizado o IMLG = peso da massa magra $(\mathrm{Kg}) /$ altura $(\mathrm{m})^{2}$, de acordo com os pontos de corte sugeridos por Kyle $^{17}$ de: $\mathrm{IMLG} \leq 17,4 \mathrm{~kg} / \mathrm{m}^{2}$ em homens e $\leq 15$ $\mathrm{kg} / \mathrm{m}^{2} \mathrm{em}$ mulheres.

\section{Análise Estatística}

Os dados foram analisados no programa Statistical Packagefor Social Siences (SPSS 23.0;IBM, Armonk, NY, EUA). Para análise de normalidade dos dados aplicamos o teste de Shapiro Wilk. Sendo assim, os dados estão apresentados em medidas de tendência central (média) e de dispersão (desvio padrão). A comparação entre as variáveis numéricas, de acordo com o estado nutricional, foi realizada por meio da análise de variância para comparações múltiplas (ANOVA) com post hoc de Tukey. Os resíduos foram avaliados segundo as suposições de normalidade, variância constante e independente. Foi considerado significativo um $\mathrm{p}<0,05$.

Tabela 1 - Características demográficas e da função pulmonar dos portadores de DPOC segundo IMC avaliados na presente pesquisa $(\mathrm{n}=27)$.

\begin{tabular}{|c|c|c|c|}
\hline Variáveis & $\begin{array}{c}\text { Desnutrição } \\
\text { n (\%) } \\
(\mathbf{n}=9)\end{array}$ & $\begin{array}{c}\text { Eutrofia } \\
\text { n (\%) } \\
(n=6)\end{array}$ & $\begin{array}{c}\text { Obesidade } \\
\text { n (\%) } \\
(\mathbf{n}=12)\end{array}$ \\
\hline \multicolumn{4}{|l|}{ Sexo } \\
\hline Masculino & $6(66,7)$ & $4(66,7)$ & $5(41,7)$ \\
\hline Feminino & $3(33,3)$ & $2(33,3)$ & $7(58,3)$ \\
\hline Idade (anos) & $65,3 \pm 7,6$ & $60,3 \pm 6,4$ & $66,2 \pm 8,98$ \\
\hline \multicolumn{4}{|l|}{ Tabagismo } \\
\hline Fumante & $2(22,2)$ & - & $3(25,0)$ \\
\hline Ex-Fumante & $7(77,8)$ & $6(100,0)$ & $9(75,0)$ \\
\hline \multicolumn{4}{|l|}{ GOLD } \\
\hline II & - & $3(50,0)$ & $3(27,3)$ \\
\hline III & $2(25,0)$ & - & $7(63,6)$ \\
\hline IV & $8(75,0)$ & $3(50,0)$ & $1(9,1)$ \\
\hline \multicolumn{4}{|l|}{ Composição Corporal } \\
\hline Massa Gorda (kg) & $12,9 \pm 3,5$ & $15,5 \pm 3,6$ & $26,0 \pm 3,5$ *\# \\
\hline \% Gordura & $24,7 \pm 7,8$ & $25,5 \pm 9,3$ & $34,6 \pm 4,6^{* \#}$ \\
\hline Massa Magra (kg) & $40,3 \pm 8,7$ & $48,2 \pm 14,6$ & $50,1 \pm 11,5$ \\
\hline \% Massa Magra & $75,2 \pm 7,8$ & $74,4 \pm 9,3$ & $65,3 \pm 4,6^{\text {*\# }}$ \\
\hline Ângulo de fase & $5,7 \pm 0,6$ & $6,0 \pm 0,8$ & $6,4 \pm 1,2$ \\
\hline $\mathrm{VEF}_{1}$ & $0,8 \pm 0,6$ & $1,4 \pm 1,0$ & $1,3 \pm 0,6$ \\
\hline$\% \mathrm{VEF}_{1}$ & $22,2 \pm 8,9$ & $43,8 \pm 27,0$ & $43,0 \pm 15,8^{*}$ \\
\hline
\end{tabular}




\section{RESULTADOS}

A idade média da amostra foi de $64,63 \pm 8,11$ anos, sendo $55,55 \%$ do sexo masculino. Observouse um predomínio de obesidade, ex-fumantes e estadiamento da doença grave, conforme estratificação da classificação do IMC, sendo nove participantes classificados em desnutrição $(33,33 \%)$ (seis homens e três mulheres), seis em eutrofia $(22,22 \%)$ (quatro homens e duas mulheres) e doze com obesidade $(44,44 \%)$ (seis homens e seis mulheres). Os indivíduos em obesidade tiveram a massa gorda (em $\mathrm{kg}$ e \%) maior do que os em eutrofia $(\mathrm{p}=0,002)$ e em desnutrição $(p=0,002)$. Já, o percentual de massa magra foi menor quando comparados aos indivíduos eutróficos $(p=0,030)$ e em desnutrição $(p=0,032)$ (Tabela 1).
A sarcopenia foi constatada em nove pacientes $(33,33 \%)$, sendo duas mulheres (GOLD IV) e sete homens (cinco classificados em GOLD IV e dois em GOLD III). Ao se estratificar esse resultado conforme o IMC, constatamos que a sarcopenia esteve presente em 20,0\% (n=8) dos nove pacientes em desnutrição e $3,70 \%(n=1)$ dos seis pacientes em eutrofia (dados não constam em tabela).

Observou-se, na análise dos micronutrientes, uma maior inadequação de consumo, com exceção da vitamina $\mathrm{E}$ nos indivíduos eutróficos. De um modo geral, os indivíduos obesos tiveram um consumo menor dos micronutrientes considerados na pesquisa, em relação aos demais grupos. Os dados referentes à média de consumo alimentar diário de macronutrientes, fibras e micronutrientes estão dispostos na Tabela 2.

Tabela 2 - Consumo alimentar médio obtido no R24h dos macronutrientes: proteína, carboidrato, lipídio (calorias); fibras (gramas) e micronutrientes ( $\mathrm{mg}$ e $\mu \mathrm{g}$ ) conforme a classificação do estado nutricional ( $\mathrm{n}=27)$.

\begin{tabular}{|c|c|c|c|}
\hline Nutrientes & Desnutrição (n=9) & Eutrofia $(n=6)$ & Obesidade $(n=12)$ \\
\hline Valor calórico diário (Kcal) & $1556,2 \pm 418,9$ & $1850,3 \pm 687,7$ & $1319,7 \pm 566,3$ \\
\hline Ácido fólico & $42,4 \pm 23,8$ & $105,1 \pm 76,7^{\alpha}$ & $37,9 \pm 36,4^{*}$ \\
\hline Adequação de ácido fólico (\%) & $13,2 \pm 7,4$ & $32,8 \pm 23,9^{\alpha}$ & $11,8 \pm 11,3^{*}$ \\
\hline Ferro & $13,5 \pm 5,2$ & $15,3 \pm 5,2$ & $11,1 \pm 4,7$ \\
\hline $\begin{array}{l}\text { Adequação de } \\
\text { ferro }(\%)\end{array}$ & $238,1 \pm 101,8$ & $272,0 \pm 88,8$ & $202,7 \pm 78,6$ \\
\hline Vit. C & $19,8 \pm 16,7$ & $64,3 \pm 43,5$ & $64,3 \pm 43,5$ \\
\hline Adequação de Vit. C (\%) & $27,6 \pm 22,1$ & $95,4 \pm 67,1$ & $69,4 \pm 74,8$ \\
\hline Vit. A & $97,0 \pm 48,6$ & $219,8 \pm 154,9$ & $120,7 \pm 122,6$ \\
\hline Adequação de Vit. A (\%) & $16,8 \pm 8,2$ & $38,2 \pm 27,5$ & $21,8 \pm 24,0$ \\
\hline Vit. E & $6,2 \pm 3,4$ & $10,8 \pm 5,5$ & $4,6 \pm 2,8^{\#}$ \\
\hline Adequação de Vit. E (\%) & $51,8 \pm 28,8$ & $90,5 \pm 46,6$ & $39,0 \pm 23,8^{\#}$ \\
\hline Cálcio & $126,5 \pm 46,0$ & $312,6 \pm 224,5^{\alpha}$ & $186,1 \pm 99,6$ \\
\hline Adequação de Cálcio (\%) & $14,4 \pm 5,8$ & $34,2 \pm 22,3^{\alpha}$ & $20,8 \pm 12,4$ \\
\hline Potássio & $1031,6 \pm 347,3$ & $1529,4 \pm 629,4$ & $984,9 \pm 481,3$ \\
\hline Adequação de Potássio (\%) & $938,3 \pm 326,1$ & $1422,6 \pm 600,6$ & $935,3 \pm 436,6$ \\
\hline Magnésio & $86,6 \pm 44,2$ & $145,1 \pm 42,3^{\alpha}$ & $84,4 \pm 43,6^{\#}$ \\
\hline Adequação de Magnésio (\%) & $26,7 \pm 13,7$ & $45,2 \pm 11,1^{\alpha}$ & $27,1 \pm 12,3^{\#}$ \\
\hline Fósforo & $591,7 \pm 253,6$ & $861,5 \pm 275,5$ & $550,5 \pm 322,5$ \\
\hline Adequação de Fósforo (\%) & $102,0 \pm 43,7$ & $148,5 \pm 47,5$ & $94,9 \pm 55,6$ \\
\hline Sódio & $884,5 \pm 379,2$ & $1481,3 \pm 640,7$ & $972,0 \pm 786,4$ \\
\hline Adequação de Sódio (\%) & $38,4 \pm 16,4$ & $64,4 \pm 27,8$ & $42,2 \pm 34,1$ \\
\hline Proteína & $18,3 \pm 4,5$ & $17,4 \pm 3,4$ & $19,3 \pm 2,9$ \\
\hline Adequação de Proteína & $122,0 \pm 30,5$ & $116,2 \pm 23,1$ & $128,8 \pm 19,8$ \\
\hline Carboidrato & $57,2 \pm 13,8$ & $54,0 \pm 7,0$ & $55,5 \pm 7,3$ \\
\hline Adequação de Carboidrato (\%) & $104,1 \pm 25,1$ & $98,2 \pm 12,7$ & $100,9 \pm 13,3$ \\
\hline Lipídio & $25,3 \pm 9,1$ & $28,5 \pm 5,4$ & $25,1 \pm 5,6$ \\
\hline Adequação de Lipídio & $84,6 \pm 30,6$ & $95,0 \pm 18,1$ & $83,7 \pm 18,9$ \\
\hline Fibras & $7,3 \pm 2,5$ & $8,9 \pm 1,4$ & $5,9 \pm 2,8$ \\
\hline Adequação de Fibras (\%) & $29,5 \pm 10,2$ & $35,6 \pm 5,8$ & $23,8 \pm 11,4$ \\
\hline
\end{tabular}

Legenda: p estatístico $<0,05$ para *Desnutrição vs Obesidade \#Eutrofia vs Obesidade ${ }^{\circ}$ Desnutrição vs Eutrofia; ANOVA com post hoc de Tukey 


\section{DISCUSSÃO}

Como principais achados do estudo destaca-se que houve predomínio de pacientes obesos em relação aos pacientes em eutrofia e em desnutrição, e no que se refere à composição corporal, pode-se constatar que o menor IMC e percentual de massa magra se relacionou com a gravidade da doença, observada na diminuição do $\mathrm{VEF}_{1}$, com diferença estatisticamente significativa. $\mathrm{Na}$ análise dos micronutrientes, de um modo geral, os indivíduos obesos tiveram um consumo menor dos micronutrientes considerados na pesquisa, em comparação aos indivíduos eutróficos e desnutridos. Observou-se níveis diminuídos da ingestão alimentar de cálcio, magnésio em todos os estados nutricionais, sendo o paciente desnutrido com pior ingestão e pior função pulmonar (GOLD IV).

No presente estudo, constatou-se um predomínio de pacientes DPOC do sexo masculino, obesos. A média de gordura total foi de $19,37 \mathrm{Kg}$, maior nos pacientes obesos em relação aos pacientes desnutridos $(p<0,05)$. A média de massa magra foi de $46,47 \mathrm{Kg}$, sendo esta menor nos pacientes obesos quando em comparação com os pacientes eutróficos e desnutridos. A maior inadequação de micronutrientes foi observada nos pacientes eutróficos em relação aos demais grupos. A sarcopenia foi observada em 33,33\% dos participantes, sendo o estado de desnutrição e pior função pulmonar (GOLD IV) o mais frequente.

A amostra foi maior de homens participantes do que mulheres, isto vem ao encontro do estudo Platino (The Latin American Project for the Investigation of Obstructive Lung Disease), que teve por objetivo verificar a prevalência de DPOC na América Latina, avaliando 1.000 indivíduos em São Paulo, 1.208 em Santiago, 1.063 na Cidade do México, 943 em Montevidéu e 1.357 em Caracas, sendo que a prevalência da DPOC também foi maior em homens, ${ }^{18}$ assim como no estudo de Menezes et al., realizado em cinco grandes cidades da América Latina: São Paulo (Brasil), Santiago (Chile), Cidade do México (México), Montevidéu (Uruguai) e Caracas (Venezuela). ${ }^{19}$

Dentre os pacientes com doença mais avançada, GOLD IV, observou-se predomínio do estado de desnutrição. Segundo a Sociedade Brasileira de Pneumologia e Tisioligia, ${ }^{2}$ ocorre predomínio do estado de desnutrição neste grupo de pacientes com uma prevalência de $22 \%$ a $24 \%$ em pacientes ambulatoriais e entre $34 \%$ e $50 \%$ em pacientes hospitalizados. Em conformidade com a literatura, o nível de gravidade das doenças pulmonares está associado com a diminuição do IMC, sendo que o baixo IMC está relacionado com alto risco de mortalidade nessa população. ${ }^{20}$

Dourado et al. ${ }^{21}$ observaram que o valor de IMC exerceu influência significativa sobre o estado de desnutrição e pior função pulmonar pode ser explicado pelo maior gasto energético, o que pode ser atribuído ao hipermetabolismo decorrente de um aumento do trabalho dos músculos respiratórios, levando a uma maior necessidade de oxigênio de portadores de DPOC. Sendo assim, podemos destacar que nossos achados vão ao encontro deste estudo. Esses músculos são submetidos a um trabalho aumentado e apresentam eficiência mecânica diminuída, devido ao aumento do trabalho ventilatório e dos mediadores inflamatórios, além da influência dos medicamentos. ${ }^{22}$

Observa-se aumento de $15 \%$ a $17 \%$ na taxa de metabolismo basal (TMB) em pacientes com DPOC. ${ }^{23}$ $\mathrm{O}$ aumento da TMB surge com maior frequência em pacientes com DPOC grave (GOLD IV), podendo levar a uma perda de peso. Fato este que pode explicar o maior consumo alimentar desta população na presente pesquisa em relação aos pacientes eutróficos e obesos.

No que concerne a massa magra na presente pesquisa, foi identificado índices maiores nos homens $(51,42 \mathrm{Kg})$, quando comparados com as mulheres $(40,20 \mathrm{Kg})$, em todas as idades. A depleção de massa muscular é o principal fator responsável pelos efeitos negativos atribuídos à desnutrição. As reservas musculares são mobilizadas para atender à demanda da síntese de proteínas nos pacientes com doenças crônicas, podendo levar a uma depleção muscular, o que representa um sério problema em pacientes com esta patologia. ${ }^{24}$ No nosso estudo, identificamos maior massa gorda nos obesos em relação aos pacientes com desnutrição e eutrofia, acompanhado de um menor percentual de massa magra.

A sarcopenia foi constatada em 33,33\%dos portadores de DPOC graves, prevalecendo este achado nos pacientes em desnutrição, o que é consistente com a literatura sobre pacientes com DPOC, na qual a prevalência relatada é de $20-40 \%{ }^{22,23}$ Esse fenômeno é um indicador da síndrome de fragilidade, a qual ocorre em aproximadamente 5 a 13\% dos indivíduos com mais de 65 anos de idade e em 20 a $40 \%$ de todos 
os pacientes com DPOC, podendo incluir ainda 10 a $15 \%$ de pacientes com DPOC que apresentam peso normal. ${ }^{24}$

Em estudo com idosos italianos, obesos e/ ou sarcopênicos, a massa muscular e massa gorda foram associadas com elevadas concentrações de proteína C reativa (PCR) e interleucina 6 (IL-6). Estes dados apoiam a evidência que a massa gorda deve ser considerada nas estimativas de prevalência de sarcopenia, sendo a obesidade um agravante da relação entre sarcopenia e inflamação. ${ }^{25}$

Outro fator que desempenha papel relevante na sarcopenia, levando a uma perda da função essencial do músculo esquelético, é o desequilíbrio entre pró-oxidantes e antioxidantes, gerando o estresse oxidativo. Este desequilíbrio se eleva em pelo menos três vezes com o envelhecimento, provocando perda de $40 \%$ da atividade da enzima Cálcio-ATPase (CaATPase) no músculo esquelético. Esta enzima atua no processo de contração-relaxamento das miofibrilas. $\mathrm{Na}$ presença de estresse oxidativo, a sensibilidade ao cálcio é alterada nas fibras musculares esqueléticas. Com a diminuição da sua atividade, a motilidade da actina fica diminuída, diminuindo a velocidade de contração-relaxamento, comumente observada no músculo esquelético de idosos. ${ }^{5}$

$\mathrm{Na}$ análise dos micronutrientes, verificou-se uma maior inadequação de consumo, com exceção da vitamina $\mathrm{E}$ nos indivíduos eutróficos. De um modo geral, os indivíduos obesos tiveram um consumo menor dos micronutrientes considerados na pesquisa, em comparação aos indivíduos eutróficos e desnutridos. Observou-se níveis diminuídos da ingestão alimentar de cálcio, magnésio em todos os estados nutricionais, sendo o paciente desnutrido com pior ingestão e pior função pulmonar (GOLD IV).

Devido aos baixos níveis de cálcio, magnésio, fósforo e potássio, a função dos músculos pode estar comprometida, em nível celular, sendo que a oferta diminuída de proteínas e fosfolipídeos também compromete a função do surfactante e pode dessa maneira contribuir para o colapso alveolar e um aumento do trabalho respiratório. ${ }^{24}$ Vale destacar que água, glicoproteínas e eletrólitos formam o muco das vias respiratórias, e que a hipoproteinemia leva ao desenvolvimento de edema pulmonar, devido à diminuição da pressão coloidosmótica, permitindo aos fluidos corpóreos movimentarem-se para o espaço intersticial. Na presente pesquisa, a ingestão de proteína, carboidratos e lipídios estavam de acordo com as recomendações diárias. ${ }^{24,26}$

Referente a adequação da ingestão de vitamina $\mathrm{C}$, que se apresentou abaixo do valor recomendado entre os pacientes desnutridos $(27,6 \%)$ e com excesso de peso $(69,4 \%)$, podemos ressaltar a importância desta, quando em deficiência, podendo afetar a síntese de colágeno, que exerce grande importância na composição do tecido conjuntivo de suporte dos pulmões. ${ }^{27} \mathrm{O}$ consumo de cigarros é um dos mecanismos envolvidos no aumento do catabolismo de proteínas na DPOC, como demonstrado em diversos estudos epidemiológicos de associação entre tabagismo e sarcopenia. ${ }^{5,26}$. Embora haja controvérsias na literatura, um estudo demonstrou associação entre carga tabágica e perda de massa magra em pacientes com DPOC, ${ }^{27}$ enquanto outros não demonstraram tal associação. ${ }^{28,29}$ No presente estudo, não foi observada associação entre carga tabágica e sarcopenia. Uma possível explicação para esse achado é devido os pacientes com DPOC apresentam um estado inflamatório elevado e persistente, com altos níveis de TNF- $\alpha$, o qual está envolvido na fisiopatologia da sarcopenia, independentemente da carga tabágica. ${ }^{27}$

No presente estudo, a frequência de pacientes obesos foi maior em relação aos pacientes em eutrofia e em desnutrição. Porém, fazendo uma análise mais profunda, utilizando-se da informação da composição corporal, pode-se constatar que o menor IMC e percentual de massa magra se relacionou com a gravidade da doença, observada na diminuição do $\mathrm{VEF}_{1}$, com diferença estatisticamente significativa. Esse fato preocupa na medida em que se encontrou também que os indivíduos em obesidade tiveram uma massa magra menor do que os eutróficos e desnutridos.

Consideramos como pontos fortes a identificação da composição corporal associada ao consumo alimentar de pacientes integrantes de um programa de reabilitação, visto que se tratam fatores importantes para qualidade de vida, e que atuam na redução de morbimortalidade. Como limitações do estudo destacamos o número amostral pequeno e a não categorização dos pacientes portadores de DPOC do tipo enfisematoso e bronquítico e uso de medicamentos associados nesta população.

\section{CONCLUSÃO}

Concluímos que as alterações na composição corporal, essencialmente na massa magra, e na presença de desnutrição se associaram com maior 
gravidade da doença pulmonar, bem como se observou consumo inadequado de micronutrientes em todos os estados nutricionais, porém mais, na presença de obesidade.

\section{REFERÊNCIAS}

1. Eagan TML, Aukrust P, Ueland T, Hardie JA, Johannessen A, Mollnes TE, Damas JK, Bakke PS, Wagner PD. Body composition and plasma levels of inflammatory biomarkers in COPD. Eur Respir J 2010;36(5):1027-1033.

2. Lorenzi G, Jardim JR, Oliveira JA, Nascimento O. II Consenso brasileiro sobre doença pulmonar obstrutiva crônica. J Bras Pneumol 2004;30(5):e421.

3. Marquis K, Debigaré R, Lacasse Y, LeBlanc P, Jobin J, Carrier G, Maltais F. Midthigh muscle cross-sectional area is a better predictor of mortality than body mass index in patients with chronic obstructive pulmonary disease. Am J of Respir Crit Care Med 2002;166(6):809-813.

4. Schols AM, Broekhuizen R, Weling-Scheepers CA, Wouters E. Body composition and mortality in chronic obstructive pulmonary disease. Am J Clin Nutr 2005;1(2):69-70.

5. Costa TMRL, Costa FM, Moreira CA, Rabelo LM, BoguszewskiCL, Borba VZC.Sarcopenia na DPOC: relação com a gravidade e o prognóstico da DPOC. J Bras Pneumol 2015;41(5):415-21. doi: http://dx.doi.org/10.1590/S180637132015000000040

6. Polkey MI, Moxham J. Attacking the disease spiral in chronic obstructive pulmonary disease. Clin Med 2006;6(2):190-6.

7. Gologanu D, Ionita D, Gartonea T, Stanescu C, Bogdan MA. Body composition in patients with Chronic Obstructive Pulmonary Disease. Maedica J Clin Med 2014;9(1):25-32.

8. GOLD. Global Initiative for Chronic Obstructive Lung Disease. Global Strategy for the Diagnoses, management, and prevention of chronic obstructive pulmonary disease updated [Internet]. 2015 [cited 2016 Oct 15]. Dispovível em: http:// goldcopd.org/

9. Gibson GJ, Whitelaw W, Siafakas N. ATS/ERS Statement on respiratory muscle testing. Am J RespirCrit Care Med 2002;166(4):521-526.

10. Pereira CA, Sato T, Rodrigues SC. New reference values for forced spirometry in white adults in Brazil. J Bras Pneumol 2007;33(4):397-406.

11. Buzzard M. 24-hour dietary recall and food record methods. In: Willett W, editor. Nutritional epidemiology. 2 nd. Oxford: Oxford University Press; 1998. p. 50-73.

12. American Academy of Family Physicians. American Dietetic Association. A physician's guide to nutrition in chronic disease management for older adults [Internet]. Washington; 2002 [cited 2016 Oct 20].Disponível em: https://innovations. ahrq.gov/qualitytools/physicians-guide-nutrition-chronicdisease-management-older-adults

13. Institute of medicine of the national academies. Dietary reference intakes for energy, carbohydrate, fiber, fat, fatty acids, cholesterol, protein, and amino acids (macronutrients) [Internet]. Washington; 2005 [cited 2016 Oct 22]. Disponível em: http://www.nap.edu/books/0309085373/html/1324.html.

14. Philippi ST. Tabela de composição de alimentos: Suporte para decisão nutricional. 2 ed. São Paulo: Manole; 2002.

15. Barents Group of KPMG Peat Marwick LLP. The clinical and cost-effectiveness of medical nutrition therapies: evidence and estimates of potential medicare savings from the use of selected nutrition intervention. Summary report prepared for the Nutrition Screening Initiative. Washington: DDC; 1996.

16. Manual de instrução Biodynamics Corporation. $1^{\mathrm{a}}$ ed. Brasil, 2007.

17. Kyle UG, Pirlich M, Lochs H, Schuetz T, Pichard C. Increased length of hospital stay in underweight and overweight patients at hospital admission: a controlled population study. Clin Nutr 2005;24(1):133-42.

18. Moreira GL, Manzano BM, Gazzotti MR, Nascimento AO, Perez-Padilla R, Menezes AMB, Jardim JR. PLATINO, estudo de seguimento de nove anos sobre DPOC na cidade de São Paulo: o problema do subdiagnóstico. J Bras Pneumol 2013;40(1):307. doi: http://dx.doi.org/10.1590/S1806-37132014000100005

19. Menezes AM, Perez-Padilla R, Jardim JR, Muinõ A, Lopez MV, Valdivia G, Montes de Oca M, Talamo C, Hallal PC, Victora CG. Chronic obstructive pulmonary disease in five Latin American cities (the Platino study): a prevalence study. Lancet 2005;366(9.500):1.875-81.

20. Celli BR, Cote CG, Marin JM, Casanova C, Montes de Oca M, Mendez RA, Plata VP, Cabral HJ.The body-mass index, airflow obstruction, dyspnea, and exercise capacity index in chronic obstructive pulmonary disease. N Engl J Med 2004;350(10):1005-12.

21. Dourado VZ, Antunes LCO, Carvalho LR, Godoy I. Influência de características gerais na qualidade de vida de pacientes com doença pulmonar obstrutiva crônica. J BrasPneumol 2004;30(3):207-14.

22. Sachs A, Lerario MC. Doenças pulmonares. In: Cuppari L, editor.Guia de nutrição clínica no adulto. São Paulo: Manole; 2002. p. 249-62.

23. Vasconcelos FC, Mota ES, Lopes MFL, Fernandes SSL, Medeiros ZL. Terapia nutricional na doença pulmonar obstrutiva crônica associada à desnutrição protéico-calórica: artigo de revisão. Rev Para Med 2002;16(1):47-52.

24. Fernandes AC, Bezerra OMPA. Terapia nutricional na doença pulmonar obstrutiva crônica e suas complicações nutricionais. J bras pneumol 2006;32(5):461-71.

25. Wagner PD. Possible mechanisms underlying the development of cachexia in COPD. Eur Respir J.2008;31(3):492501.

26. Rom O, Kaisari S, Aizenbud D, Reznick AZ. Sarcopenia and smoking: a possible cellular model of cigarette smoke effects on muscle protein breakdown. Ann N Y Acad Sci 2012;1259:4753.

27. Eagan TML, Gabazza EC, D’Alessandro-Gabazza C, GilBernabe P, Aoki S, Hardie JA, Bakke PS, Wagner PD. TNF- $\alpha$ is associated with loss of lean body mass only in already cachectic 
COPD patients. Resp Res 2012;13(48):01-10.

28. Tanni SE, Pelegrino NRG, Angeleli AYO, Correa C, Godoy I. Smoking status and tumor necrosis factor-alpha mediated systemic inflammation in COPD patients. J Inflamm (Lond) 2010;7(29):01-07.

29. Iwaniec UT, Fung YK, Cullen DM, Akhter MP, Haven MC, Schmid M. Effects of nicotine on bone and calciotropic hormones in growing female rats. Calcif Tissue Int 2000;67(1):68-74.

Como citar: BAELZ, Katiele et al. Aspectos nutricionais na doença pulmonar. Revista Interdisciplinar de Promoção da Saúde, Santa Cruz do Sul, v. 2, n. 3, set. 2020. ISSN 2595-3664. Disponível em: <https://online.unisc.br/seer/index.php/ripsunisc/article/view/14073>. Acesso em: 02 jul. 2019. doi:https://doi.org/10.17058/rips.v2i3.14073 